\title{
Imidazolium-based functional monomers for the imprinting of the anti-inflammatory drug naproxen: Comparison of acrylic and sol-gel approaches
}

\author{
Porkodi Kadhirvel $^{\mathrm{a}, *}$, Manuel Azenha ${ }^{\mathrm{a}, *}$, Sudhirkumar Shinde ${ }^{\mathrm{b}}$, Eric Schillinger ${ }^{\mathrm{b}}$, \\ Paula Gomes ${ }^{a}$, Börje Sellergren ${ }^{b}$, A. Fernando Silva ${ }^{a}$ \\ a CIQ-UP, Departamento de Química e Bioquímica, Faculdade de Ciências, Universidade do Porto, Portugal \\ b INFU, Faculty of Chemistry, Technical University of Dortmund, Otto-Hahn-Str. 6, D-44221 Dortmund, Germany
}

\section{A R T I C L E I N F O}

\section{Article history:}

Received 4 July 2013

Received in revised form 3 September 2013

Accepted 4 September 2013

Available online 9 September 2013

\section{Keywords:}

Molecular imprinting

Cationic monomer

Sol-gel

Methacrylic polymer

Naproxen

Frontal analysis

\begin{abstract}
A B S T R A C T
Imidazolium-based monomers were, for the first time, employed in a comprehensive investigation of the molecular imprinting process of naproxen in both acrylic and sol-gel tridimensional networks. To this end, molecularly imprinted polymer (MIP) and xerogel (MIX) were both optimized for performance, by testing different porogen, template speciation and component ratios. The developed imprints were characterized for their pore properties (nitrogen adsorption analysis), site heterogeneity, binding properties and other performance parameters such as the imprinting factor, selectivity (HPLC column tests), column efficiency and mass transfer kinetics (frontal analysis study). MIP exhibited mesoporosity $\left(D_{p}\right.$ $29 \mathrm{~nm}$ ), whereas MIX did not, which was reflected in both the lower number of accessible imprinted sites $(4.9 \mu \mathrm{mol} / \mathrm{g}$ versus $3.7 \mu \mathrm{mol} / \mathrm{g}$ ) and the slower binding/dissociation in MIX. The naproxen/ibuprofen selectivity ratio was estimated as 6.2 for the MIX and 2.5 for the MIP. Given the high importance of capacity and fast mass transfer in typical applications of imprinted materials, and the satisfactory selectivity of MIP, it can be concluded that the acrylic approach was globally the most advantageous. Still, the remarkably high selectivity of MIX and its reasonable capacity demonstrate that future work devoted to further optimization of both formats is worthwhile.
\end{abstract}

(C) 2013 Elsevier B.V. All rights reserved.

\section{Introduction}

Molecular imprinting is a versatile technique for preparing synthetic materials with tailored molecular recognition properties; as such, is presently attracting widespread interest, especially in chromatography, (bio)chemical sensing, drug delivery and catalysis [1]. The preparation of molecularly imprinted polymers (MIP) typically involves three steps: first, a monomer-template [M-T] complex is formed via self-assembly; next, the M-T complex is polymerized with an excess of cross-linker to form a rigid polymer; finally, the template is removed, leaving behind binding cavities which are complementary in shape and functional group to the template [2]. The vast majority of MIP is based on the use of organic acrylate-type polymers: a standard procedure using a methacrylate monomer, with a nearly optimal ratio to the template molecule and crosslinker, is described in numerous works [2-8]. The broad applicability of methacrylic acid (MAA) as a functional monomer

\footnotetext{
* Corresponding authors. Tel.: +351 220402628; fax: +351220402659.

E-mail addresses: porkodikathirvel@yahoo.com (P. Kadhirvel), mazenha@fc.up.pt (M. Azenha).
}

in MIP production is related to the fact that the carboxylic acid group serves well as a hydrogen bond and proton donor as well as a hydrogen bond acceptor [9]. Nevertheless, a common severe constraint of this type of imprinting is the need for an organic solvent in which all species are soluble. This further precludes use of template molecules that are only soluble in aqueous media, which implies obvious limitations in the development of MIP for many environmental and biological applications. Although some promise has been shown for aqueous-based rebinding procedures, such results are rare and considerable progress is required to overcome this limitation $[10,11]$.

Sol-gel imprinting is one possible alternative for achieving molecular recognition of hydrophilic targets, given the ease of preparation of sol-gels and their compatibility with polar environments [12]. Sol-gel imprinting is straightforward and provides an efficient way for preparing hybrid matrices through incorporation of organic components into inorganic polymers under mild thermal conditions, while controlling MIP thickness, porosity and surface area. However, to date, amorphous sol-gel molecularly imprinted xerogels (MIX) have not demonstrated the same degree of success as MIP for analytical applications, namely in the form of bulk materials. 
A minimal number of reports $[10,13]$ regarding comparison of acrylic and sol-gel systems can be found in the literature. Cummins et al. [10], compared the bulk acrylic and sol-gel systems for the imprinting of 2-aminopyridine; these authors replicated the acrylic MIP synthesized by Zhou and He [14], while used commercially available monomers as phenyltrimethoxysilane (PTMOS) and aminopropyltrimethoxysilane (APTES) for the synthesis of the sol-gel MIX. By comparing the performance of both imprinted materials in different solvents, Cummings et al. concluded that the acrylic polymers exhibited high selectivity with poor affinity in chloroform, whereas the sol-gel MIX had the opposite behavior, i.e., exhibited high affinity and poor selectivity. In acetonitrile and methanol, however, both materials behaved similarly. In another work, Marx et al. [13], used propanolol as model template for the comparison of the acrylic and sol-gel systems; they prepared MIP and MIX as films that were tested for their enantiomeric separation ability. The acrylic system was prepared using the typical polymerization mixture of MAA and ethyleneglycoldimethacrylate (EDMA), whereas PTMOS was used as the functional monomer for the sol-gel approach. The authors found that the acrylic system exhibited high propranolol uptake, but this was accompanied by a high degree of non-specific binding in aqueous buffer. In contrast, the sol-gel system displayed lower uptake, but remarkably lower nonspecific binding. The uptake kinetics of the acrylic polymer was significantly slower than that of the sol-gel polymer. The imprinting of the sol-gel film with enantiomerically pure $(S)$-propranolol resulted in its pronounced chiral recognition over the $(R)$-enantiomer, while no information related with the enantiomeric recognition in acrylic polymer was provided.

The above unique published examples of research dealing with comparison of acrylic and sol-gel approaches to produce molecularly imprinted materials suggest that the latter might successfully challenge the dominance of the former. In view of this, a deeper study of acrylic versus sol-gel molecular imprinting approaches is justified. Hence, we herein report the first of a series of studies concerning the thorough study of sol-gel and acrylic imprinting approaches for selected templates, representative of important chemical families. A comprehensive, systematic and detailed study of the molecular imprinting of naproxen (NAP), a profen-like nonsteroidal anti-inflamatory drug (NSAID), is described, embracing pre-polymerization issues (such as choosing a common functional group), microstructure, thermal stability, selectivity and affinity properties, as well as mass transfer kinetics.

\section{Materials and methods}

\subsection{Chemicals}

\subsubsection{Sol-gel approach}

Ureidopropyltrimethoxysilane (UPTMOS), the template (+)(S)-2-(6-methoxynaphthalen-2-yl) propanoic acid (S-NAP) and trifluoroacetic acid (TFA) were obtained from Sigma-Aldrich, Germany. The sodium salt of naproxen (Na-NAP) was prepared by adding aqueous $\mathrm{NaOH}$ to S-NAP in equimolar amounts, followed by solvent removal. 2-(2-pyridylethyl trimethoxysilane) (PETMOS), 3-iodopropyltrimethoxysilane and $N$-(3-triethoxysilylpropyl)-4,5dihydroimidazole were from ABCR, Germany, and used as received. Formic acid, tetramethoxysilane (TMOS), methyltrimethoxy silane (MTMOS), chlorotrimethoxysilane and 1,1,1,3,3,3hexamethylsilanedisilazane were purchased from Fluka, Germany. Tetrahydrofuran (THF), acetonitrile and methanol (MeOH) of HPLC grade were obtained from VWR International. All other reagents used were of analytical grade. Millipore water of Milli-Q quality (Millipore, Italy) was used.

\subsubsection{Acrylic approach}

1-Allyl-3-vinylimidazolium bromide (IL-A) was obtained from Shanghai Cheng Jie Chemical Co. Ltd., China. Ethylene glycol dimethacrylate (EDMA) was obtained from Sigma-Aldrich, Germany. EDMA was purified by washing consecutively with $10 \%$ aqueous $\mathrm{NaOH}$, water, brine, and finally water; after drying over $\mathrm{MgSO}_{4}$, pure, dry EDMA was obtained by distillation under reduced pressure. The initiator $N, N^{\prime}$-azo-bis-(2,4-dimethyl)valeronitrile (ABDV) was purchased from Wako Chemicals, Germany. Dry chloroform, acetonitrile, tetrahydrofuran and methanol were from Fluka, Germany, and used as received.

\subsection{Synthesis of cationic sol-gel functional monomer}

This synthesis is represented in Fig. 1. 1-(triethoxysilylpropyl)3-(trimethoxysilylpropyl)-4,5-dihydroimidazolium iodide (IL-SG) was synthesized by adapting a literature procedure [15]. Hence, $\mathrm{N}$-(3-triethoxysilylpropyl)-4,5-dihydroimidazole (2.74 g, $10 \mathrm{mmol}$ ) and (3-iodopropyl)trimethoxysilane $(2.97 \mathrm{~g}, 10 \mathrm{mmol})$ were dissolved in $10 \mathrm{~mL}$ of dry acetonitrile. The mixture was refluxed for $14 \mathrm{~h}$ under nitrogen. Excess acetonitrile was distilled off using rotary evaporator and the resulting product was isolated as a lightbrown liquid. Product's characterization by ${ }^{1} \mathrm{H}$ NMR, ${ }^{13} \mathrm{C}$ NMR and LC-MS, allowed to obtain data (see below) in perfect agreement with both the expected structure and previously published NMR data for the same compound [15].

${ }^{1} \mathrm{H} \mathrm{NMR}\left(400 \mathrm{MHz}, \mathrm{CDCl}_{3}\right): \delta 0.38\left\{\mathrm{~m}, 4 \mathrm{H}-\mathbf{C H}_{\mathbf{2}} \mathrm{Si}-\left(\mathrm{OCH}_{2} \mathrm{CH}_{3}\right)_{3}\right.$ and $\left.\left.\left(\mathrm{CH}_{3}\right)_{3} \mathrm{SiCH}_{2}-\right)\right\}, \delta 0.96\left(\mathrm{t}, 9 \mathrm{H}-\mathrm{SiOCH}_{2} \mathrm{CH}_{3}\right), \delta 1.53\{\mathrm{~m}, 4 \mathrm{H}$, $-\mathbf{C H}_{2} \mathrm{CH}_{2} \mathrm{Si}\left(\mathrm{OCH}_{2} \mathrm{CH}_{3}\right)_{3}$ and $\left.\left(\mathrm{CH}_{3} \mathrm{O}\right)_{3} \mathrm{SiCH}_{2} \mathbf{C H}_{2}-\right\}, \delta 3.3\{\mathrm{~s}, 9 \mathrm{H}$ $\left.\left(\mathbf{C H}_{3} \mathrm{O}\right) 3 \mathrm{Si}-\right\}, \delta 3.4\left\{\mathrm{~m}, 4 \mathrm{H}=\mathrm{NCH}_{2} \mathrm{CH}_{2} \mathrm{~N}=\right.$ and $\left.=\mathrm{NCH}_{2} \mathbf{C H}_{2} \mathrm{~N}=\right\}$, $\delta 3.5\left\{\mathrm{q}, 6 \mathrm{H},-\mathrm{Si}-\mathrm{OCH}_{2} \mathrm{CH}_{3}\right)_{3}, \delta 3.8\left\{\mathrm{t}, 4 \mathrm{H},=\mathrm{NCH}_{2} \mathrm{CH}_{2}-\right.$ and $\left.-\mathrm{CH}_{2} \mathbf{C H}_{2}=\mathrm{N}-\right\}, \delta 8.8\{\mathrm{~s}, 1 \mathrm{H},=\mathrm{NCHN}=) .{ }^{13} \mathrm{C}$ NMR Data (in $\left.\mathrm{CDCl} 3\right)$ $\delta 6.1$ and $7.3\left[-\mathrm{CH}_{2} \mathrm{Si}\left(\mathrm{OCH}_{2} \mathrm{CH}_{3}\right)_{3}\right.$ and $\left.\left(\mathrm{CH}_{3} \mathrm{O}\right)_{3} \mathrm{SiCH}_{2}-\right], 18.4$ $\left(-\mathrm{SiOCH}_{2}-\mathrm{CH}_{3}\right), \quad 20.9$ and $21.2\left[-\mathrm{CH}_{2} \mathrm{CH}_{2} \mathrm{Si}\left(\mathrm{OCH}_{2} \mathrm{CH}_{3}\right)_{3}\right.$ and $\left(\mathrm{CH}_{3} \mathrm{O}\right)_{3} \mathrm{SiCH}_{2} \mathrm{CH}_{2}-\mathrm{]}, 48.4$ and $48.5 \quad\left(=\mathrm{NCH}_{2} \mathrm{CH}_{2} \mathrm{~N}=\right.$ and $\left.=\mathrm{NCH}_{2} \mathrm{CH}_{2} \mathrm{~N}\right), 50.2$ and $50.3\left(=\mathrm{NCH}_{2} \mathrm{CH}_{2}-\right.$ and $\left.-\mathrm{CH}_{2} \mathrm{CH}_{2} \mathrm{~N}\right)$, $50.9\left[\left(\mathrm{CH}_{3} \mathrm{O}\right)_{3} \mathrm{Si}-\right], 58.7\left[-\mathrm{Si}\left(\mathrm{OCH}_{2}-\mathrm{CH}_{3}\right)_{3}, 157.8(=\mathrm{NCHN}=)\right.$. MS $(\mathrm{ESI}+\mathrm{MeOH}) \mathrm{m} / \mathrm{z}$ : calculated 437.38 and found 437.38 .

\subsection{UV-vis spectrophotometric analysis of pre-polymerization solutions}

A series of template-monomer solutions was prepared by adding different amounts of IL-SG (3) or IL-A (4) (Fig. 2) to a fixed concentration of $0.5 \mathrm{mmol} / \mathrm{L}$ S-NAP in methanol or chloroform, respectively. The concentration of the cationic monomers was varied from 0 to $4.5 \mathrm{mmol} / \mathrm{L}$. The change in the absorbance of the solutions was scanned by UV-vis spectrophotometry in the 200-1800 nm range, against the relevant cationic monomer solutions (i.e., lacking S-NAP) as references.

\subsection{Synthesis of xerogels and polymers}

A series of sol-gel imprinted and non-imprinted xerogels (MIX or NIX, respectively) was prepared using PETMOS (1), UPTMOS (2), or IL-SG (3) functional monomers (Fig. 2). In all MIX, the template to functional monomer, functional monomer to crosslinker, and silane to water content ratios were maintained as $1: 2,1: 6.6$, and $1: 2$, respectively. The synthesis of IL-SG derived MIX is described below (Section 2.4.1), whereas detailed procedures for the synthesis of other xerogels prepared (compositions as given in Table S1) are provided as Supporting Information.

Imprinted and non-imprinted methacrylic polymers (MIP or NIP, respectively) were also prepared using the ionic liquid-based organic functional monomer IL-A (4), as described in Section 2.4.3. 
<smiles>COS(CCCI)(OC)OC</smiles>

(3-iodopropyl)trimethoxyxilane<smiles>CCOC(CC)(CCCC1CCNC1)OCC</smiles>

1-(3-(triethoxysilyl)propyl)-4,5-dihydro-1H-imidazole<smiles>CCOS(CCCN1CCN(CCC[As](OC)(OC)OCC)C1[18F])(OCC)OCC</smiles>

(1-(triethoxysilylpropyl), 3-(trimethoxysilylpropyl)-4,5-dihydroimidazolium iodide)

Fig. 1. Scheme of the synthesis of the sol-gel cationic (IL-SG) precursor.<smiles>COC(O)(O)CCc1ccncc1</smiles>

(1) PETMOS

4-(2-Pyridyl)ethyltrimethoxysilane<smiles>CO[Si](CCCI)(OC)OC</smiles>

(3) IL-SG

1-(triethoxysilylpropyl)-3-(trimethoxysilylpropyl) -4,5-dihydroimidazolium iodide<smiles>COc1ccc2cc([C@@H](C)C(=O)O)ccc2c1</smiles>

(5) S-NAP<smiles>CCO[Si](CCCNC(N)=O)(OCC)OCC</smiles>

(2) UPTMOS

1-[3-(Trimethoxysilyl)propyl]urea<smiles></smiles>

(4) IL-A

1-Allyl-3-vinylimidazolium bromide<smiles>CC(C)Cc1ccc(C(C)C(=O)O)cc1</smiles>

(6) IBU

Fig. 2. (1-4) Structure of functional monomers tested for the imprinting of S-NAP; (5-6): structures of S-NAP and its structural analogue IBU.

The formulation of pre-polymerization mixtures for imprinted and non-imprinted materials is summarized in Table 1.

\subsubsection{Synthesis of xerogels using the cationic IL-SG functional} monomer

IL-SG (423 mg, $0.75 \mathrm{mmol})$, Na-NAP (94.5 mg, $0.375 \mathrm{mmol}$ ) and $735 \mu \mathrm{l}(5 \mathrm{mmol})$ of TMOS were mixed together in a $50 \mathrm{~mL}$ centrifuge tube, and the mixture dissolved in $\mathrm{MeOH}(11.6 \mathrm{~mL})$, followed by water $(202 \mu \mathrm{L})$ addition (see Table 1$)$.
The centrifuge tube was then sealed with parafilm and punctured with small holes, allowing for slow evaporation of solvent. The mixture stirred using a magnetic stirrer with pellet, until the gel was formed. It was then dried in open air atmosphere.

\subsubsection{Endcapping of surface silanol groups in xerogels}

Half of the available amount of dry MIX/NIX xerogels was endcapped by alkylation of unreacted free $-\mathrm{OH}$ groups, whereas the other half was left as non-endcapped to ascertain the influence

Table 1

Prepolymerization solution composition used to prepare the sol-gel (MIX/NIX) and methacrylic polymers (MIP/NIP).

\begin{tabular}{|c|c|c|c|c|c|}
\hline Polymer/Xerogel & Template & Functional monomer & Crosslinker & Porogen & Catalyst or initiator \\
\hline MIX & Na-NAP (0.37 mmol) & IL-SG (0.75 mmol) & TMOS (5 mmol) & $\mathrm{MeOH}(11.6 \mathrm{~mL})$ & Water $(102 \mu \mathrm{L})$ \\
\hline NIX & - & IL-SG (0.75 mmol) & TMOS (5 mmol) & $\mathrm{MeOH}(11.6 \mathrm{~mL})$ & Water $(102 \mu \mathrm{L})$ \\
\hline MIP & S-NAP $(0.37 \mathrm{mmol})$ & IL-A $(0.75 \mathrm{mmol})$ & EDMA (7.5 mmol) & $\mathrm{CHCl}_{3}: \mathrm{MeOH}(2 \mathrm{~mL}: 0.8 \mathrm{~mL})$ & ABDV \\
\hline NIP & - & IL-A (0.75 mmol) & EDMA (7.5 mmol) & $\mathrm{CHCl}_{3}: \mathrm{MeOH}(2 \mathrm{~mL}: 0.8 \mathrm{~mL})$ & ABDV \\
\hline
\end{tabular}


of endcapping on the recognition efficiency in xerogels. In the endcapping process, the xerogel was treated with an equimolar mixture of chlorotrimethylsilane $(2.5 \mathrm{~g}, 23 \mathrm{mmol})$ and 1,1,1,3,3,3hexamethyldisilazane $(3.8 \mathrm{~g}, 23 \mathrm{mmol})$ at room temperature, for $24 \mathrm{~h}$. The xerogel was then washed with THF and acetonitrile to remove excess endcapping reagents [16].

\subsubsection{Metacrylic polymer synthesis}

Metacrylic imprinted polymer (MIP) was prepared using S-NAP as template, IL-A (4) as functional monomer, EDMA as crosslinker and $\mathrm{CHCl}_{3}$ as porogen.

Thus, S-NAP ( $230 \mathrm{mg}, 1 \mathrm{mmol})$, IL-A ( $860 \mathrm{mg}, 4 \mathrm{mmol})$, EDMA ( $3772 \mu \mathrm{L}, 20 \mathrm{mmol}$ ) and initiator ABDV ( $1 \% \mathrm{w} / \mathrm{w}$ of total monomers) were dissolved in $\mathrm{CHCl}_{3}(5.6 \mathrm{~mL})$. The solution was transferred to a glass ampoule, cooled to $0{ }^{\circ} \mathrm{C}$ and purged with a flow of dry nitrogen for $5 \mathrm{~min}$. The tube was then flame-sealed while still under cooling, and the polymerization initiated by placing the tubes in a thermostatic water bath pre-set at $50^{\circ} \mathrm{C}$. After $24 \mathrm{~h}$, the tubes were broken and the polymers lightly crushed.

\subsubsection{Template removal}

A preliminary template extraction from lightly crushed xerogels, either endcapped (EC) or non-endcapped (NEC), and polymers, was done on a Soxhlet apparatus, using $10 \%$ formic acid in methanol. The option for coarse particles at this stage allowed for higher yields of material. Washing was continued until the extracted liquid phase showed undetectable levels of S-NAP by reverse-phase HPLC with UV detection at $230 \mathrm{~nm}$. The materials were then crushed in a mortar and sieved to select two fractions of particles with sizes ranging $25-45$ and $45-75 \mu \mathrm{m}$, respectively. The larger $45-75 \mu \mathrm{m}$ particles were used in solid phase extraction (SPE) cartridges, whereas the smaller 25-45 $\mu$ m ones were used for packing HPLC columns. Final removal of template was then accomplished by extensive percolation of the packed particles with $10 \%$ formic acid in methanol.

\subsection{Material characterization}

Surface micrographs of the prepared materials were acquired using a Hitachi H-S4500 FEG Microscope in secondary electron mode, with an acceleration voltage of $1 \mathrm{kV}$. The samples were deposited on holders with a carbon foil without gold sputtering.

Thermogravimetric analysis was carried out using a TGAQ50 (TA instruments, Eschborn, Germany). Each sample (10-15 mg) was placed in a platinum pan, which was suspended in a sensitive balance together with the reference pan. The sample was then heated in a furnace at a heating rate of $20^{\circ} \mathrm{C} / \mathrm{min}$, under $\mathrm{N}_{2}$ atmosphere.

The surface area and the pore parameters were determined on a Quantachrome Autosorb 6B (Quantachrome Corporation, Boynton Beach, FL) automatic adsorption instrument. Prior to measurements, $100-150 \mathrm{mg}$ of the samples were heated at $40-60^{\circ} \mathrm{C}$ under high vacuum (10-5 Pa) for at least $12 \mathrm{~h}$. The specific surface areas $(S)$ were evaluated using the BET method, the specific pore volumes $\left(V_{p}\right)$ following the Gurvitch method and the average pore diameter $\left(D_{p}\right)$ using the BJH theory applied to the desorption branch of the isotherm.

\subsection{Solid phase extraction}

SPE cartridges were packed with $200 \mathrm{mg}$ of the S-NAP imprinted (45-75 $\mu \mathrm{m})$ and the corresponding non-imprinted xerogels. The cartridges were conditioned with $5 \mathrm{~mL}$ of water, and a sample containing 3 ppm S-NAP in $\mathrm{MeOH}(1 \mathrm{~mL})$ was percolated at a constant flow rate of $0.5 \mathrm{~mL} / \mathrm{min}$, in a Visiprep (Supelco, Bellefonte, USA) SPE station manifold. After sample loading, $1 \mathrm{~mL}$ of water was used as the washing solution. The cartridges were thereafter subjected to an elution step by percolating $1 \mathrm{~mL}$ of $5 \%$ formic acid in $\mathrm{MeOH}$. The elution fractions (loading, washing and elution) from the SPE column were directly monitored by reversed phase HPLC (HewlettPackard HP 1050 instruments, Agilent Technologie, Waldbronn, Germany) with a Nucleosil ODS $\mathrm{C}_{8}$ column $(25 \times 0.46 \mathrm{~cm})$ with $5 \mu \mathrm{m}$ particles. The mobile phase, $2 \%$ acetic acid in $70: 30(\mathrm{v} / \mathrm{v})$ $\mathrm{MeOH} / \mathrm{H}_{2} \mathrm{O}$, was flowed through the column at $0.65 \mathrm{~mL} / \mathrm{min}$ and the detection performed by UV absorbance reading at $230 \mathrm{~nm}$. The resulting peak areas were used to calculate the amount of sorbentbound analyte. Each data point was based on the average of two replicate measurements and the recovery \% was calculated according to Eq. (1).

Recovery $(\%)=100-\frac{[\text { Load }]-[\text { Eluate }]}{[\text { Load }]} \times 100$

Regeneration of the SPE cartridge was achieved by reconditioning with $5 \mathrm{ml}$ of $\mathrm{MeOH}$ and reequilibrating with $5 \mathrm{~mL}$ of water.

\subsection{Chromatographic evaluation}

The smaller-sized particles of the prepared materials were typically slurry-packed into stainless steel columns $(50 \times 4.6 \mathrm{~mm})$, using $\mathrm{MeOH} / \mathrm{H}_{2} \mathrm{O} 80: 20(\mathrm{v} / \mathrm{v})$ as pushing solvent, for evaluation of their chromatographic performance. Pure methanol with 0.05 or $0.1 \%$ acetic acid was used as a mobile phase for xerogels and polymers, respectively. The flow rate was fixed as $0.5 \mathrm{~mL} / \mathrm{min}$ if not otherwise mentioned. Aliquots $(20 \mu \mathrm{L})$ of $10 \mathrm{ppm}$ solutions of SNAP and $30 \mathrm{ppm}$ of ibuprofen (IBU) in methanol were injected, and elution monitored at $230 \mathrm{~nm}$.

The retention factor $(k)$, the separation factor $(\alpha)$ and the imprinting factor (IF) were calculated using the following formulae $k=\left(t-t_{0}\right) / t_{0} ; \alpha=k_{\text {nap }} / k_{\mathrm{ibu}}$ and $\mathrm{IF}=k_{\text {mip }} / k_{\text {nip }}$, where $t$ is the retention time of the template S-NAP or of its analogue IBU, and $t_{0}$ is the retention time of the void marker.

The column efficiency has been demonstrated in terms of number of theoretical plates $(N)$, which was calculated according to Eq. (2).

$N=5.545\left(\frac{\tau R}{W_{h}}\right)$

where $N$ is the number of theoretical plates, $t_{R}$ is the retention time and $W_{h}$ is the peak width at half height (in time units).

The binding properties of the materials were determined by classical staircase frontal analysis (for a proper understanding of the technique, reading of references $[17,18]$ is suggested). For this purpose, stock solutions of S-NAP or IBU were prepared in $0.05 \%$ acetic acid in $\mathrm{MeOH}$ at two different concentrations: 0.05 and $0.5 \mathrm{mmol} / \mathrm{L}$. These solutions were used as mobile phase components at different percentages, mixing with pure mobile phase $(0.05 \%$ acetic acid in $\mathrm{MeOH})$, and thus allowing gradient elution. All experiments were carried out at room temperature and $0.5 \mathrm{~mL} / \mathrm{min}$ flow rate, with detection at $230 \mathrm{~nm}$, following the order of increasing concentration without washing cycles in between the staircases. At the end of the experiments, the column was flushed for $1 \mathrm{~h}$ at $1 \mathrm{~mL} / \mathrm{min}$ using $5 \%$ formic acid in $\mathrm{MeOH}$. For a series of $\mathrm{n}$ successive steps, the sample concentration in the stationary phase in step $n\left(q_{n}\right)$, at equilibrium with concentration $C_{n}$ in the mobile phase, was calculated using the integral mass balance equation (Eq. (3)).

$q_{n+1}=\frac{q_{n}+\left(C_{n+1}-C_{n}\right) F_{V}\left[t_{\mathrm{eq}}-\left(t_{0}-t_{\mathrm{ea}}\right)-t_{\mathrm{ep}}\right]}{\left[V_{c}-F_{v}\left(t_{0}-t_{\mathrm{ea}}\right)\right.}$

where $F_{v}$ is the flow rate, $t_{0}$ is the measured void time of the column, $t_{\mathrm{ea}}$ and $t_{\mathrm{ep}}$ are the extra-column times (from injector and pump, respectively) determined by replacing the column with a zero dead volume connector. $t_{\text {ea }}$ was determined by injecting from 
the sampler, and $t_{\mathrm{ep}}$ by running a step gradient with subsequent determination of breakthrough times. $V_{c}$ is the geometrical volume of the column tube.

\subsection{Isotherm fitting}

Non-linear fitting of theoretical isotherms to experimental data was performed using Origin 5.0. The adsorption isotherm models commonly evaluated for imprinted materials are Langmuir (Eq. (4)), Freundlich (Eq. (5)) and Langmuir-Freundlich, L-F (Eq. (6)).

$q=\frac{q * K C}{1+K C}$

$q=a C^{m}$

$q=\frac{q *(K C)^{m}}{1+(K C)^{m}}$

where $q$ is the concentration in the stationary phase at equilibrium with concentration $C$ in the mobile phase. The Langmuir model assumes that one class of site is present on the surface, with saturation capacity $q^{*}$ and dissociation constant $K$. The Freundlich isotherm, on the other hand, assumes sites with a Gaussian distribution of binding strengths. Here the width of the Gaussian distribution describes the degree of heterogeneity, through the index $m$.

The difference between the L-F model and the Freundlich one is evident at high sorbate concentrations, for which the L-F model is able to represent the saturation behaviour. At low sorbate concentrations, the L-F equation reduces to the classical Freundlich equation. On the other hand, as $m$ approaches unity, indicative of a completely homogeneous sorbent surface (i.e., energetic equivalence of all binding sites), the L-F equation reduces to the classical Langmuir equation. Thus, the hybridized L-F isotherm is able to model adsorption of solutes at high and low concentrations onto both homogeneous and heterogeneous sorbents.

The estimates of the modelled parameters are given at the asymptotic 95\% confidence interval. For each model and each set of experimental data, the Fisher parameter was calculated according to the following equation (Eq. (7)).

$F_{\text {calc }}=\frac{m-l \sum_{i=1}^{n}\left(q_{\mathrm{exp}, i}-q_{\mathrm{exp}}\right)^{2}}{m-1 \sum_{i=1}^{n}\left(q_{\mathrm{exp}, i}-q_{t, i}\right)^{2}}$

where $q_{\text {exp }, i}$ are the experimental values of the solid-phase concentration of the adsorbate for a given system, $q_{\exp }$ is the mean value of the data set, $q_{\exp , i}$, for a given system, $q_{t, i}$ is the estimate of the solid-phase concentration of the adsorbate by a given model, $l$ is the number of adjusted parameters in the model, and $\mathrm{m}$ is the number of experimental data-points for a given system [19].

An additional model, the Hill-coefficient, was considered. This model assumes that the template molecules bind to the sorbent in a cooperative manner [20]. The sorbent is assumed to bind to $n$ ligands simultaneously, $\mathrm{n}$ being determined from the slope of the following linear relation (Eq. (8)):

$\log \left[\frac{q}{(1-q)}\right]=n \cdot \log C-\log K$

where $\theta$ stands for the ratio $q / q^{*}$.

\section{Results and discussion}

\subsection{The common functional monomer issue}

The fundamental condition to carry out a meaningful comparative study of sol-gel and acrylic approaches toward the preparation of MIM is to ensure that functional monomers used in both approaches are as similar as possible, hence establishing basically the same interactions with the template molecule. However, to the best of our knowledge, this is the first report where such monomer similarity was a primary concern. The search for a suitable functional monomer common to both approaches began with the pyridine core, on the basis of previous works where methacrylic imprints for S-NAP were prepared using vinyl pyridine as functional monomer [21-24].

In view of the above, a commercially available siloxane functional monomer bearing the pyridine ring, PETMOS (1), was tested for the sol-gel process. Expectedly, PETMOS should be capable of a strong acid-base interaction with the acidic end of the NAP's carboxylic group, while possibly benefiting from the solvatophobic effect of the water/MeOH mixture, contributing to the overall stabilization of the M-T complex. Evaluation of the xerogels' performance by SPE allowed concluding that all those deriving from PETMOS had a negligible MIX to NIX difference in sorption behaviour in the course of loading, washing and all eluting steps performed (see Fig. S1 for a typical result). Therefore, no imprinting behaviour was observed, indicating the essentially non-specific nature of the binding sites in these xerogels. In view of this, UPTMOS (2) was regarded as a possibly better alternative, expected to form a stable M-T complex through multiple hydrogen bonding interactions between the template's carboxyl and the monomer's terminal urea group $\left(-\mathrm{HN}-\mathrm{CO}-\mathrm{NH}_{2}\right)$. However, unsatisfactory results were also obtained in this case (data not shown), suggesting that a stronger M-T interaction, such as ionic pairing, would be needed. Consequently, it was hypothesized that the imidazolium moiety from IL-SG would interact more strongly with the S-NAP's carboxylate, allowing to improve the selectivity properties of the imprinted xerogel. Likewise, the same should apply if the analogous cationic functional monomer, IL-A (4), was used in the preparation of MIP trough the acrylic approach.

As a preliminary verification of the above expectations, UV-vis studies were undertaken to evaluate the interactions between IL-SG (3) or IL-A (4) and S-NAP (detailed spectral data are supplied as Supporting Information). Hence, addition of the functional monomers to a fixed concentration of S-NAP resulted in the observation that an increasing concentration of either IL-SG or IL-A provoked a bathochromic shift of the absorbance maxima of S-NAP, in the range $270-280 \mathrm{~nm}$, and an overall decrease in band intensity. This corroborates the formation of M-T complexes between S-NAP and the monomers, similarly to previous observations on strong interactions occurring between 4-vinylpyridine (4-VP) and S-NAP as template molecule [24]. Still, while this effect could be observed when using the siloxane monomer (IL-SG) in methanol, a combination of methanol with a much less polar solvent, such as chloroform, was necessary to obtain a similar effect with the acrylic counterpart, IL-A. These results were consistent with those obtained following several trials of MIP synthesis comprising different porogens, from polar like methanol to weakly polar such as chloroform, with varying crosslinkers, component ratios, and template speciation (used as either the neutral or anionic form). These trials showed that an imprinting effect was only obtained when using weakly polar solvents associated to the neutral form of the template. Sol-gel preliminary tests, on the contrary, showed that better results emerged from associating a polar porogen with the anionic form of the template. Consequently, different porogens had to be used depending on the format (polymer versus xerogel), hence sacrificing porogen uniformity for the sake of optimal imprinting effects.

In addition, the UV-vis spectra allowed to roughly estimate the optimum ratio of the functional monomer to template as being 4 for the acrylic and 2 for the sol-gel system, since beyond those ratios there was negligible change in the intensity and wavelength shift of absorbance maxima. 

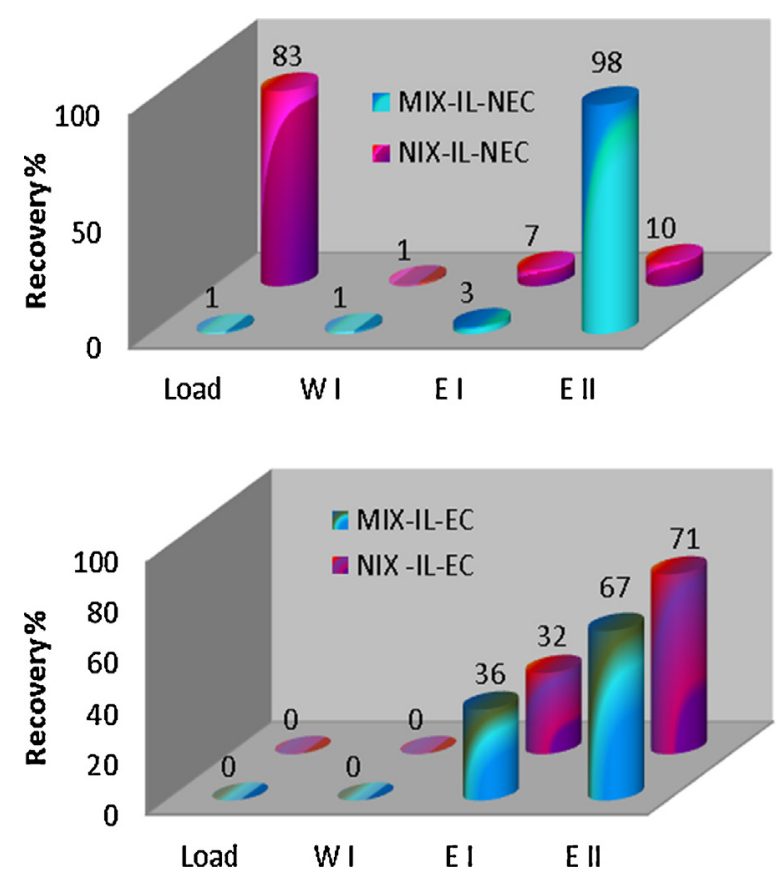

Fig. 3. SPE result of MIX/NIX-IL-NEC (non endcapped) and MIX/NIX-IL-EC (endcapped) xerogels. SPE steps: load: 3 ppm S-NAP in methanol - $1 \mathrm{~mL}, \mathrm{~W}$ I: water $1 \mathrm{~mL}, \mathrm{E} \mathrm{I}: \mathrm{MeOH}-1 \mathrm{~mL}$, E II:5\% formic acid in methanol $-1 \mathrm{~mL}$.

Imprinted and non-imprinted xerogels, and polymers, have been synthesized from mixtures reflecting the presented preliminary outcomes. A quick SPE evaluation of the non-endcapped (NEC) and end-capped (EC) sol-gel materials (MIX-IL and NIX-IL) was conducted to ascertain the usefulness of the end-capping procedure, as shown in Fig. 3. Concerning non-endcapped xerogels, it was clearly demonstrated that MIX-IL-NEC had much higher affinity, with a retention of $c a .100 \%$ for 3 ppm S-NAP load (in $\mathrm{MeOH}$ ), and could be easily and completely eluted with $5 \%$ formic acid in $\mathrm{MeOH}$; in turn, about $85 \%$ of S-NAP was recovered while loading in NIX-IL-NEC, and the remaining was eluted with water washing and elution steps. This constituted a promising result concerning the imprinting of S-NAP in MIX prepared using IL-SG.

End-capped xerogels showed different retention behaviour in SPE as compared to their non-endcapped counterparts; namely, both MIX-IL-EC and NIX-IL-EC retained $c a$. $100 \%$ of loaded S-NAP (in $\mathrm{MeOH}$ ) and similar recovery in all subsequent steps. This behaviour may be explained by the non-specific hydrophobic interaction exerted by the insertion of $-\mathrm{CH}_{3}$ groups inherent to the endcapping process, resulting in the masking of the less numerous or less accessible imprinted sites. Hence, it was decided to keep the non-end-capped xerogels for the remaining of the study, despite the potential problems arising from the presence of residual silanol groups in the network, such as non-selective binding and network additional shrinkage due to ageing (additional condensation within the gel).

\subsection{Comparative pore structure and thermal stability}

Imprinted polymers and xerogels, prepared using cationic functional monomers IL-A and IL-SG, respectively, were characterized by TGA, BET and SEM. The BET specific surface area $(S)$, specific pore volume $\left(V_{p}\right)$ and average pore diameter $\left(D_{p}\right)$ were calculated from the nitrogen adsorption isotherms and \% mass loss was observed from TGA, as described in the experimental part. The values concerning the pore properties are collected in Table 2. It is observed that the methacrylic polymers exhibited large BET surface area ( $c a$. $320 \mathrm{~m}^{2} / \mathrm{g}$ ) while for the sol-gel materials it was negligible. The
Table 2

Pore properties of the imprinted and non-imprinted methacrylic and sol-gel materials.

\begin{tabular}{llll}
\hline Sample & \multicolumn{3}{l}{ BET } \\
\cline { 2 - 4 } & $S\left(\mathrm{~m}^{2} / \mathrm{g}\right)$ & $D_{p}(\mathrm{~nm})$ & $V_{p}(\mathrm{~mL} / \mathrm{g})$ \\
\hline MIX & 2.4 & 3.0 & 0.0038 \\
NIX & ND & 4.9 & 0.0013 \\
MIP & 313 & 29 & 0.55 \\
NIP & 334 & 29 & 0.58 \\
\hline
\end{tabular}

ND, non-determined.

average pore diameter $\left(D_{p}\right)$ of $29 \mathrm{~nm}$ obtained for the methacrylic polymer clearly indicates its mesoporous nature. In contrast, the xerogels appear to be essentially microporous, which is not conveniently dealt with by use of the BET technique $[25,26]$. Such difference in porosity is distinctly seen from the SEM images in Fig. S4, which clearly show that MIP is highly porous (mesoporosity), whereas MIX presents a dense structure suggesting microporosity.

No or little difference was found between imprinted and non-imprinted materials, either polymers or xerogels, concerning surface areas or pore volume and diameter. This indicates that the presence of the template molecule did not influence the porous network of the resulting materials.

The mesoporous nature of methacrylic polymers with pore size $\left(D_{p}\right)$ of $29 \mathrm{~nm}$ is expected to provide easier access to the inner imprinted cavities, as compared to microporous xerogels $\left(D_{p}<2 \mathrm{~nm}\right)$ where the diffusion is expectedly hindered. For most applications in liquid media, permanent porosity and a large surface area of accessible meso and macropores are preferred [27].

The TGA diagrams of imprinted and non-imprinted methacrylic and sol-gel materials may be found as Supporting Information (Fig. S5), and demonstrate that the two types of material display distinct thermal decomposition profiles. Hence, $c a .100 \%$ decomposition and weight loss began at around $250^{\circ} \mathrm{C}$ for both imprinted and non-imprinted polymers. In turn, for MIX and NIX xerogels, slow degradation proceeds from 100 to $400{ }^{\circ} \mathrm{C}$, followed by a sudden $32 \%$ mass loss at around $400^{\circ} \mathrm{C}$. Hence, the presence of the organic moieties (-propyl dehydroimidazolium) in the hybrid xerogel caused a slow degradation starting at a relatively low temperature. Although the initial mass loss in xerogels starting around $100{ }^{\circ} \mathrm{C}$ is probably due to the release of unreacted species or non-removed template, it appears from the TGA results that the methacrylic system is thermally more stable up to around $250^{\circ} \mathrm{C}$. Unfortunately, TGA data gives information about mass loss from the materials, but not about eventual imprinting site disruption, which should occur earlier than mass loss. It would be helpful to compare glass transition temperatures for both materials, which was actually attempted by differential scanning calorimetry (DSC) analysis. But, as often occurs in this type of DSC analysis [28], the glass transition peaks were too weak to be distinguished from baseline noise. Future evaluation of thermal disruption of imprinted sites may eventually pass by exposing the imprinted materials to successively increasing temperatures, followed by assessment of the binding performance (affinity, selectivity) after exposure at each temperature level.

\subsection{Comparative chromatographic evaluation of imprinted materials}

\subsubsection{Selectivity and imprinting performance}

Methacrylic and sol-gel materials were packed in small columns and assessed by liquid chromatography for their ability to separate the template S-NAP from its structural analogue IBU (Fig. 2). IBU was chosen as a reference instead of R-NAP, after concluding that none of the materials was able to separate the enantiomers despite the many chromatographic conditions tested (data not shown). 

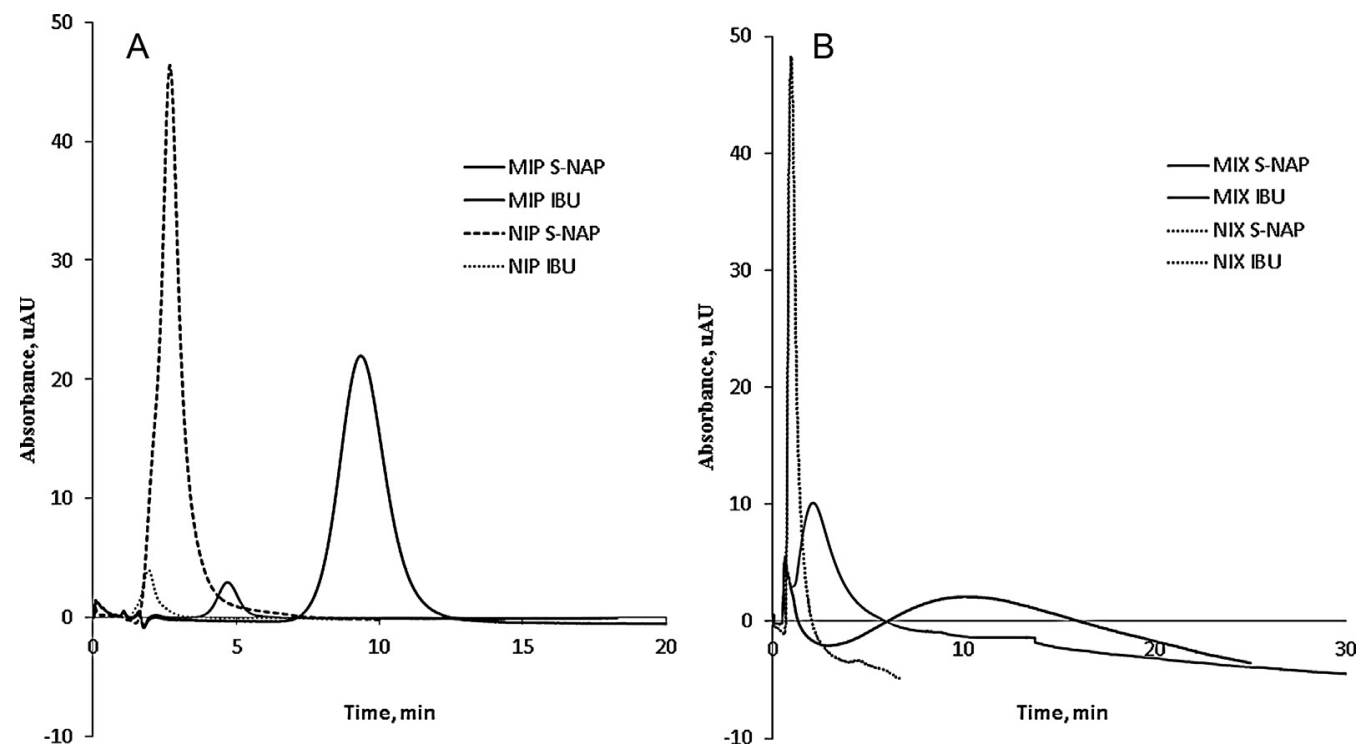

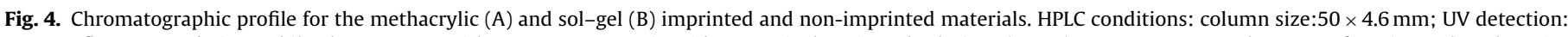

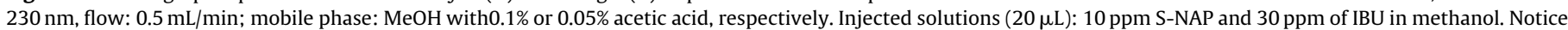
the superposed elution profiles of S-NAP and IBU with the NIX material.

In order to calculate the separation factor $(\alpha)$ and imprinting factor (IF), methanolic solutions of S-NAP and IBU were injected into the packed columns, yielding the chromatograms shown in Fig. 4. Retention times were noted from the chromatographic peaks, and the calculated values of $\alpha$ and IF of the materials obtained are presented in Table 3.

It is evident from Fig. 4A that the imprinted polymer showed higher retention for the template S-NAP than for its structural analogue IBU ( $\alpha=2.5$ ), proving the imprinting behaviour. The nonimprinted polymer (NIP) also discriminated the template and its analogue, but to a markedly lesser extent ( $\alpha=1.1$ ), possibly due to non-specific interactions related to the mesoporous nature and functionalization of the material [27].

On the other hand, imprinted and non-imprinted xerogels performed more distinctly. Fig. 4B clearly shows that the imprinted xerogel had a much higher affinity for S-NAP than for its structural analogue IBU $(\alpha=6.23)$, which demonstrates the highly specific recognition of the imprinted template. In turn, the non-imprinted xerogel was unable to discriminate the template and its analogue $(\alpha=1)$.

The efficiency of the prepared HPLC columns, which in practice relates to the width of the chromatographic peak observed at a certain retention time, indirectly provides information about the mass transfer kinetics between the supports and the templates, and may also reflect the degree of heterogeneity of the sorption sites. Theoretical number of plates of 6 and 125 were estimated according to Eq. (2) for MIX and MIP, respectively, demonstrating the much higher column efficiency obtained for MIP.

In summary, the imprinted xerogel showed markedly better selectivity than its polymer counterpart, but with very low column efficiency. In turn, the methacrylic MIP presented much better column efficiency associated to reasonable selectivity. The dissimilar efficiencies seem to indicate that the quality of template imprinting is much higher in the sol-gel approach, which is unfortunately eclipsed by its microporous nature. In contrast, methacrylic materials present better mass transfer kinetics and/or less heterogeneous binding site distribution, eventually associated to their mesoporous nature.

\subsubsection{Sorption isotherm analysis}

To get deeper insight into the methacrylic and sol-gel materials binding properties such as, capacity, affinity constant, heterogeneity index and mass transfer kinetics, frontal analysis was conducted. Such analysis allowed to estimate the amount of template, or its analogue, adsorbed $(q)$ onto the imprinted and non-imprinted stationary phases in equilibrium with a given mobile phase concentration, $C$, using Eq. (3).

Data points $(q, C)$ obtained (Fig. 5$)$ were then fitted to the different isotherm models, such as Langmuir (Eq. (4)), Freundlich (Eq. (5)) and hybrid Langmuir-Freundlich (Eq. (6)) by non-linear regression analysis. Since some of the isotherms appeared to follow a sigmoidal pattern, the Hill coefficient model was also checked against the experimental data-set by using the linearization Eq. (8). A sigmoidal shape may be related to cooperative binding phenomena where binding to the sorbent becomes facilitated (positively cooperative binding) or hampered (negatively cooperative binding) by previously adsorbed ligands.

The complete fitted data-set is presented as Supporting Information. Table 4 comprises the most relevant data, namely those obtained from the model that fitted best to the experimental data for each material and sorbate. The MIP-associated data-set was the only one fitting the Hill coefficient model, as shown in Fig. S6,

Table 3

Chromatographic properties of methacrylic and sol-gel material towards S-NAP and IBU.

\begin{tabular}{|c|c|c|c|c|c|}
\hline \multirow[t]{2}{*}{ Materials } & \multirow[t]{2}{*}{ Retention factor, $k_{\mathrm{NAP}}$} & \multirow[t]{2}{*}{ Retention factor, $k_{\mathrm{IBU}}$} & \multirow[t]{2}{*}{ Selectivity $\alpha e l e k_{\mathrm{NAP}} / k_{\mathrm{IBU}}$} & \multicolumn{2}{|c|}{ Imprinting factor $=k_{\mathrm{MIP}} / k_{\mathrm{NIP}}$} \\
\hline & & & & IBU & S-NAP \\
\hline MIX & 32.40 & 5.20 & 6.2 & 3.9 & 24 \\
\hline NIX & 1.35 & 1.35 & 1.0 & & \\
\hline MIP & 7.03 & 2.86 & 2.5 & 3.6 & 7.7 \\
\hline NIP & 1.00 & 0.90 & 1.1 & & \\
\hline
\end{tabular}


Table 4

Selected isotherm fitting parameters.

\begin{tabular}{|c|c|c|c|c|c|c|}
\hline & Sorbate & Model & $K\left(\mathrm{~L} \mathrm{mmol}^{-1}\right)$ & $q^{\mathrm{a}}(\mu \mathrm{mol} / \mathrm{g})$ & $m$ & $n$ \\
\hline \multirow[t]{2}{*}{ MIP } & S-NAP & $\mathrm{HC}^{\mathrm{a}}$ & $8.5^{\mathrm{b}}$ & 4.9 & - & 1.4 \\
\hline & IBU & $\mathrm{HC}^{\mathrm{a}}$ & $40^{\mathrm{b}}$ & 2.2 & - & 1.6 \\
\hline \multirow[t]{2}{*}{ NIP } & S-NAP & $\mathrm{LF}$ & 100 & 2.8 & 1.0 & - \\
\hline & IBU & LF & 100 & 3.6 & 0.86 & - \\
\hline \multirow[t]{2}{*}{ MIX } & S-NAP & $\mathrm{F}^{\mathrm{a}}$ & 250 & 3.7 & 0.58 & - \\
\hline & IBU & $\mathrm{F}^{\mathrm{a}}$ & 111 & 0.60 & 0.44 & - \\
\hline \multirow[t]{2}{*}{ NIX } & S-NAP & $\mathrm{F}^{\mathrm{a}}$ & 71 & 0.32 & 0.37 & - \\
\hline & IBU & $\mathrm{F}^{\mathrm{a}}$ & 48 & 0.36 & 0.32 & - \\
\hline
\end{tabular}

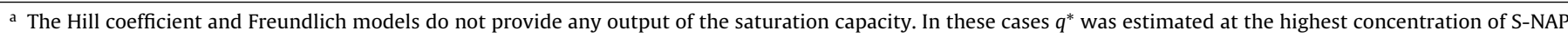
and IBU used.

b Binding constants values obtained by the HC model have $\left(M^{-n}\right)$ units, hence are not directly comparable.

meaning that a positively cooperative binding mechanism $(n>1)$ was likely active during the sorption process. Possible mechanisms explaining cooperativity in MIP are: (i) formation of highly ordered complexes such as (template $)_{n}$-(functional monomer $)_{m}$ in pre-polymerization mixtures, resulting in imprinted sites for the (template $)_{n}$ complexes whose abundance in the rebinding solution will increase with increasing concentration [29]; (ii) induced fit, which refers to a process where the initial interaction between the material and the ligand is relatively weak, but which rapidly induces conformational changes in the structure of the material that strengthen binding; induced fit has typically been related to highly swelling systems for protein imprinting [30] and to covalently imprinted polymers [31]. Such mechanism is thus unlikely affecting the present S-NAP imprinted polymer. A capable elucidation of this question is beyond the scope of this study, and depends on future focused work. In any case, whatever mechanism was active here, seems also to accommodate IBU rebinding, another interesting feature deserving further study, which is in line with the lower selectivity found in the frame of MIP/MIX comparison.
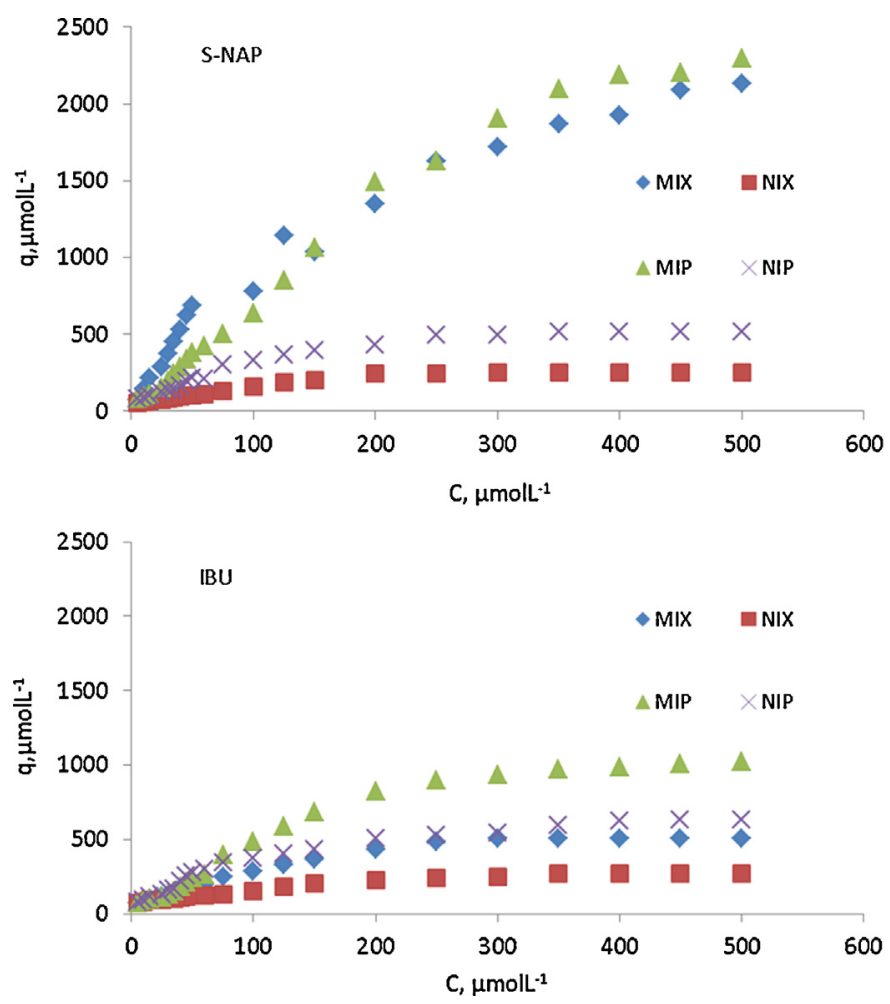

Fig. 5. Equilibrium binding isotherms for S-NAP and IBU as determined from staircase frontal analysis of the methacrylic (MIP and NIP) and sol-gel (MIX and NIX) materials. $q$ : sorbate concentration in the tested material; $C$ : concentration of the sorbate present in the mobile phase.
Both the aforementioned cooperative binding mechanisms require the presence of imprinted sites; therefore, the NIPassociated data-set did not fit the Hill coefficient model, as expected. Instead, it best fitted the L-F model. The saturation capacities $\left(q^{*}\right)$ of the imprinted and non-imprinted polymers were estimated for the template, S-NAP: ca. 4.9 and $2.8 \mu \mathrm{mol} / \mathrm{g}$, respectively. For its analogue IBU, the respective values were $c a .2 .2$ and $3.6 \mu \mathrm{mol} / \mathrm{g}$. The imprinted methacrylic polymer exhibited thus a better capacity for the template S-NAP, roughly two times higher than that for the structural analogue IBU. Also significantly higher capacity towards S-NAP was observed the MIP relatively to NIP, reinforcing the conclusion that a satisfactorily imprinted polymer was produced.

Some considerations about site heterogeneity can also be drawn. In the case of MIP, site homogeneity is an assumption of the model which is corroborated by the goodness of fit observed. In the case of NIP, a heterogeneity parameter was calculated from the L-F fitting, the material exhibiting homogeneous binding sites as indicated by " $m$ " value close to 1 . The symmetric peak obtained in chromatogram (Fig. 4A) for S-NAP with the MIP/NIP materials agrees with these data.

In what regards sol-gel materials, different adsorption behaviour was observed, as it was found that the Freundlich model allowed for the best fit of xerogel-associated data-sets. The estimated saturation capacity, $\mathrm{q}^{*}$, for MIX showed a much higher value for S-NAP, $3.7 \mu \mathrm{mol} / \mathrm{g}$, than for IBU, $0.60 \mu \mathrm{mol} / \mathrm{g}$. Furthermore, a much higher affinity was found for S-NAP $\left(K=250 \mathrm{~L} \mathrm{mmol}^{-1}\right)$ over IBU $\left(K=111 \mathrm{~L} \mathrm{mmol}^{-1}\right)$. Both $q^{*}$ and $K$ values for MIX were much higher than those for NIX, confirming the excellent imprinting effect on MIX. Approximate values of $m=0.5$

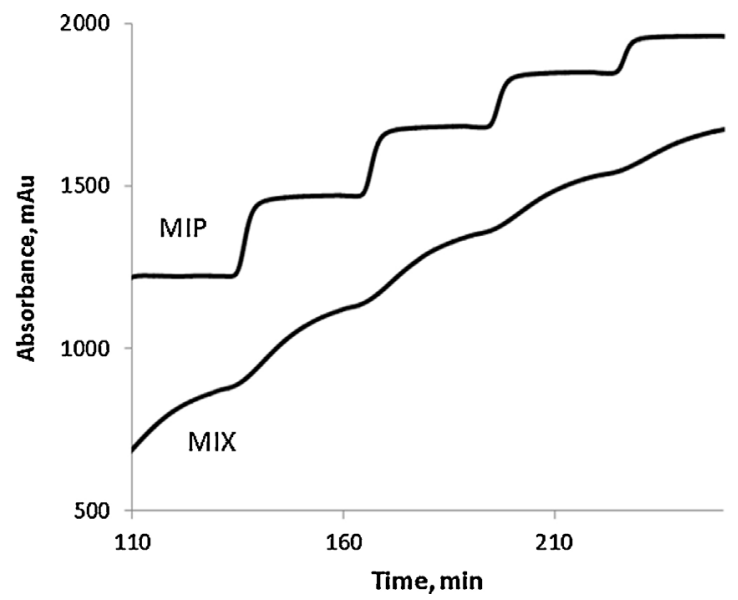

Fig. 6. Frontal chromatograms (partial view) obtained for the sol-gel (MIX) and methacrylic (MIP) imprinted materials, allowing the observation of a much faster stabilization of the detector signal for the MIP after each change in the concentration of S-NAP in the mobile phase. 
indicated site distribution heterogeneity. This is in agreement with the pronounced peak broadening and tailing (Fig. 4B) obtained in the MIX-packed HPLC column. Still, peak broadening may also be partially explained by the poor mass transfer properties associated with the microporous structure of MIX (Table 2). Taken together, frontal analysis results allow to state that MIP exhibited higher capacity and more homogeneous binding sites that MIX.

Finally, another interesting observation was obtained from frontal chromatograms, which agreed well with BET, SEM and HPLC analysis already described. As clearly seen in Fig. 6, MIP reached equilibrium much faster than MIX, irrespective of flow rate and concentration. For example, for the $5-10 \mu \mathrm{M}$ concentration step, the equilibration time required by MIP was $7.7 \mathrm{~min}$, while for MIX 16.80 min were needed. Again, this may be explained by the absence of mesoporosity in MIX, which in fact hinders an easy flow, resulting in longer equilibration time.

\section{Conclusion}

The main objective of this work was to compare the two different synthetic approaches and get important insights in terms of material pore properties, performance parameters such as imprinting factor and selectivity, and binding properties including binding capacity and affinity, heterogeneity index, column efficiency and mass transfer kinetics. Stemming from highly similar cationic functional monomers targeting the carboxylic group in the template, MIP and MIX materials were optimized for performance, implicating that different porogen, template speciation and component ratios had to be used for the two approaches. Such functional grouporiented study of course does not mean that better imprints in a particular format could not be obtained with other functional groups, as evidenced for the methacrylic imprint produced with 4-VP, which exhibited a superior selectivity (enantioseparation) as compared to those reported here. It is therefore essential to keep relativity in mind when following the next considerations, which concern comparison of the two imprinting processes studied.

Pore structure plays an important role in the performance of imprints, and a very important difference between the two networks in this regard emerged. In fact, MIP exhibited mesoporosity and MIX did not, what constituted a serious handicap for MIX performance, as reflected in terms of diminished accessible imprinted sites (capacity) and of slower binding/dissociation kinetics. Globally we can state that the sol-gel approach allowed for higher selectivity and imprinting factor, while the methacrylic polymerization approach was superior in terms of capacity and mass transfer kinetics of the produced imprinted material. The higher selectivity found for MIX may be partially attributable to the solvent memory effect (rebinding tested in methanol), but other factors may be involved, such as not having used exactly the same functional group and template speciation in both approaches. Besides high selectivity, a good capacity and fast kinetics are highly desirable features in the application of imprinted materials such as an imprinted stationary phase in chromatography. Since MIP presented a quite satisfactory selectivity, all considered, it seems reasonable to conclude that, within the constraints inherent to the development of this comparative work, the acrylic approach was the most successful.

It is our conviction that future developments in the sol-gel procedure towards mesoporosity, for example by using macromolecular templating, may accomplish significant improvement in MIX performance. On the other hand, a deeper understanding of factors underlying the apparent cooperative binding mechanisms active for both S-NAP and IBU with MIP may help devising modified procedures resulting in an improved selectivity. One final word regarding use of imidazolium-based cationic functional monomers: this virtually unexplored functionality, in the context of imprinting processes, appears to be rather interesting and possibly advantageous for the imprinting of other templates capable of assuming anionic or highly electron-donating forms.

\section{Acknowledgements}

The authors thank Fundação para a Ciência e Tecnologia (FCT), Lisbon, Portugal, and to FEDER for financial support (PEstC/QUI/UI0081/2011). P.K. thanks FCT also for the Ph.D. Grant SFRH/BD/44715/2008.

\section{Appendix A. Supplementary data}

Supplementary data associated with this article can be found, in the online version, at http://dx.doi.org/10.1016/j.chroma.2013. 09.015 .

\section{References}

[1] J. Wang, P.A.G. Cormack, D.C. Sherrington, E. Khoshdel, Pure Appl. Chem. 79 (2007) 1505

[2] Y. Zhang, D. Song, L.M. Lanni, K.D. Shimizu, Macromolecules 43 (2010) 6284.

[3] B. Sellergren, B. Ekberg, K. Mosbach, J. Chromatogr. 347 (1985) 1.

[4] B. Sellergren, J. Wieschemeyer, K.S. Boos, D. Seidel, Chem. Mater. 10 (1998) 4037.

[5] M.-J. Syu, T.-C. Chiu, C.-Y. Lai, Y.-S. Chang, Biosens. Bioelectron. 22 (2006) 550.

[6] Y. Li, X. Li, Y. Li, C. Dong, P. Jin, J. Qi, Biomaterials 30 (2009) 3205.

[7] F. Rong, P. Li, High Perform. Polym. 23 (2011) 585.

[8] M.R. Halhalli, E. Schillinger, C.S.A. Aureliano, B. Sellergren, Chem. Mater. 24 (2012) 2909.

[9] M.H. Abraham, P.P. Duce, D.V. Prior, D.G. Barratt, J.J. Morris, P.J. Taylor, J. Chem. Soc. Perk. Trans. 12 (1989) 669

[10] W. Cummins, P. Duggan, P. McLoughlin, Anal. Chim. Acta 542 (2005) 52.

[11] B. Dirion, Z. Cobb, E. Schillinger, L.I. Andersson, B. Sellergren, J. Am. Chem. Soc. 125 (2003) 15101

[12] S. Fireman-Shoresh, S. Marx, D. Avnir, Adv. Mater. 19 (2007) 2145.

[13] S. Marx, Z. Liron, Chem. Mater. 13 (2001) 3624.

[14] J. Zhou, X.W. He, Anal. Chim. Acta 381 (1999) 85.

[15] B. Lee, H.J. Im, H.M. Luo, E.W. Hagaman, S. Dai, Langmuir 21 (2005) 5372.

[16] C.I. Lin, A.K. Joseph, C.K. Chang, Y.C. Wang, Y.D. Lee, Anal. Chim. Acta 481 (2003) 175.

[17] S.G. Guiochon, A.M. Katti, Fundamentals of Preparative and Nonlinear Chromatography, Academic Press, Boston, MS, 1994.

[18] I. Quinones, G. Guiochon, J. Chromatogr. A 796 (1998) 15.

[19] H.J. Kim, F. Gritti, G. Guiochon, J. Chromatogr. A 1049 (2004) 25.

[20] A.V. Hill, Proc. Phys. Soc. (1910) iv.

[21] M. Kempe, K. Mosbach, J. Chromatogr. A 664 (1994) 276.

[22] C. Baggiani, C. Giovannoli, L. Anfossi, C. Passini, P. Baravalle, G. Giraudi, J. Am. Chem. Soc. 134 (2013) 1513.

[23] Y. Duana, C. Daia, Y. Zhang, L. Chen, Anal. Chim. Acta 758 (2013) 93.

[24] Y. Lu, C.X. Li, H.S. Zhang, X.H. Liu, Anal. Chim. Acta 489 (2003) 33.

[25] C. Herdes, L. Sarkisov, Langmuir 25 (2009) 5352.

[26] L. Guardia, R. Badia-Laino, M.E. Diaz-Garcia, C.O. Ania, J.B. Parra, Biosens. Bioelectron. 23 (2008) 1101

[27] B. Sellergren, Man-Made Mimics of Antibodies and their Applications in Analytical Chemistry, Elsevier, Amsterdam, The Netherlands, 2001.

[28] A. Bachmann, Advances in Light Curing Adhesives, P. SPIE, San Diego, CA, 2001.

[29] J. Svenson, J.G. Karlsson, I.A. Nicholls, J. Chromatogr. A 1024 (2004) 39.

[30] W. Lei, Z. Meng, W. Zhang, L. Zhang, M. Xue, W. Wang, Talanta 99 (2012) 966.

[31] G. Wulff, Chem. Rev. 102 (2002) 1. 